TRANSACTIONS OF THE

AMERICAN MATHEMATICAL SOCIETY

Volume 364, Number 8, August 2012, Pages 4283-4301

S 0002-9947(2012)05547-4

Article electronically published on March 29, 2012

\title{
AN APPROACH TO HIGHER ORDER LINKING INVARIANTS THROUGH HOLONOMY AND CURVATURE
}

\author{
JAMES J. HEBDA AND CHICHEN M. TSAU
}

\begin{abstract}
We study the Milnor-Massey linking invariants through the holonomy and curvature of certain nilpotent connections and their flat quotient connections. Versions of the Porter-Turaev Theorem are proved in the context of de Rham cohomology.
\end{abstract}

\section{INTRODUCTION}

This article applies the theory of connections from differential geometry to prove de Rham cohomology versions of the Porter-Turaev Theorem which correlate the Milnor and Massey invariants of a link in the 3-sphere [12, 18]. We have borrowed several ideas from [11, specifically how to realize Massey products as the curvature of certain nilpotent connections and how the Milnor numbers figure into the holonomy of a longitude. Nonetheless, we present a counterexample in $\S 4.2$ to two of its theorems. We skirt these problems with a different approach that hinges on calculating the full holonomy of the commutator of a longitude and meridian, rather than, as in [11, the holonomy of a longitude alone in a subconnection. Systematic use of flat quotient connections facilitates our holonomy calculations. A well-known generalized Gauss-Bonnet formula, which expresses the holonomy along a contractible loop in terms of a curvature integral, provides the basic relation between the Massey and Milnor numbers. This Gauss-Bonnet formula, which can be quite complicated due to noncommutativity, simplifies considerably in the present case because the curvature lies in the center of a nilpotent Lie algebra on which the associated Lie group acts trivially.

The link between holonomy (or parallel transport) and curvature has a history that goes back to Levi-Civita in an infinitesimal form [9]. Schlesinger [15] found a formula for the holonomy along a closed contractible loop in terms of a curvature integral which is valid for connections in vector bundles. He also showed it to be a generalization of the classical Gauss-Bonnet formula on surfaces. More generally, such formulas are valid in Chen's theory of formal connections, where they appear as a type of Stoke's Theorem for the loop space of a differentiable manifold [4]. Chen also noted the theory of formal connections could be used to study the invariants of links. (See also [6].)

Received by the editors September 25, 2009 and, in revised form, December 15, 2010. 2010 Mathematics Subject Classification. Primary 57M25; Secondary 53C05, 57M27.

Key words and phrases. Nilpotent connection, holonomy, curvature, Milnor numbers, Massey product, Porter-Turaev Theorem.

(C)2012 American Mathematical Society Reverts to public domain 28 years from publication 


\section{Nilpotent CONNECTIONS}

Let $n$ be a positive integer. The associative algebra of $(n+1) \times(n+1)$ real upper triangular matrices will be denoted $\mathcal{A}$. Let $\mathfrak{J}$ denote the subset of $\mathcal{A}$ of strictly upper triangular matrices, that is, with zeroes on the diagonal. Then $\mathfrak{J}$ is a nilpotent ideal in $\mathcal{A}$ with $\mathfrak{J}^{n+1}=\{0\}$. The group of upper triangular matrices with 1 's along the main diagonal, the so-called unitriangular matrices, will be denoted by $G$. In other words,

$$
G=\{I+X: X \in \mathfrak{J}\},
$$

where $I$ denotes the $(n+1) \times(n+1)$ identity matrix. Then $G$ is a nilpotent Lie group whose Lie algebra can be identified with $\mathfrak{J}$ under the usual bracket product of matrices

$$
[X, Y]=X Y-Y X \quad \text { for } \quad X, Y \in \mathfrak{J} .
$$

Moreover, for $I+X \in G$, one has the following formula for the group inverse:

$$
(I+X)^{-1}=I-X+X^{2}-X^{3}+\cdots+(-1)^{n} X^{n} .
$$

Notation. The nonzero entries of a matrix $X$ in $\mathfrak{J}$ will be indexed by sequences $r \ldots s$ of consecutive integers from $r$ through $s$ inclusive, assuming $r \leq s$. With this convention the entry $X_{r \ldots s}$ lies in the $r$-th row and the $(s+1)$-st column of $X$.

Let $E^{r \ldots s}$ denote the matrix in $\mathfrak{J}$ all whose entries are 0 except the $(r \ldots s)$-th entry, which equals 1 . Obviously the set $\left\{E^{r \ldots s}\right\}$ forms a vector-space basis for $\mathfrak{J}$. Their products satisfy

$$
E^{p \ldots q} E^{r \ldots s}=\left\{\begin{array}{cl}
E^{p \ldots s} & \text { if } r=q+1, \\
0 & \text { otherwise. }
\end{array}\right.
$$

A connection in the trivial principal $G$-bundle

$$
\pi: M \times G \rightarrow M
$$

over a smooth manifold $M$ is defined by giving a $\mathfrak{J}$-valued 1 -form $\omega$ on the base manifold $M$. Connections of this form play a special role in [7]. We can write $\omega$ in the matrix form

$$
\omega=\left(\begin{array}{ccccc}
0 & \omega_{1} & \omega_{12} & \cdots & \omega_{12 \ldots n} \\
0 & 0 & \omega_{2} & \cdots & \omega_{2 \ldots n} \\
\vdots & \vdots & \vdots & \ddots & \vdots \\
0 & 0 & 0 & \cdots & \omega_{n} \\
0 & 0 & 0 & \cdots & 0
\end{array}\right)=\sum_{r \ldots s} \omega_{r \ldots s} E^{r \ldots s}
$$

where each of the entries $\omega_{r . . s}$ of the matrix are 1 -forms on $M$.

Remark 2.0.1. By [8, Proposition 1.4, p. 66], there exists a unique connection form $\hat{\omega}$ on the principal bundle $M \times G$ that satisfies $\sigma^{*}(\hat{\omega})=\omega$, where $\sigma: M \rightarrow M \times G$, $\sigma(x)=(x, I)$, is the canonical global section.

2.1. Curvature and Massey products. The curvature of the connection $\omega$ is the $\mathfrak{J}$-valued 2 -form $\Omega$ on $M$ defined by the structure equation:

$$
\Omega=d \omega+\omega \wedge \omega .
$$


This is just the structure equation $d \hat{\omega}+\hat{\omega} \wedge \hat{\omega}=\hat{\Omega}$ on the bundle [8, p. 77] pulled down to the base by the canonical section. We can write $\Omega$ in the matrix form

$$
\Omega=\left(\begin{array}{ccccc}
0 & \Omega_{1} & \Omega_{12} & \cdots & \Omega_{12 \ldots n} \\
0 & 0 & \Omega_{2} & \cdots & \Omega_{2 \ldots n} \\
\vdots & \vdots & \vdots & \ddots & \vdots \\
0 & 0 & 0 & \cdots & \Omega_{n} \\
0 & 0 & 0 & \cdots & 0
\end{array}\right)=\sum_{r \ldots s} \Omega_{r \ldots s} E^{r \ldots s},
$$

where each of the entries of the matrix are 2-forms on $M$. By taking exterior derivatives we obtain the Bianchi identity:

$$
d \Omega=\Omega \wedge \omega-\omega \wedge \Omega .
$$

The $n$-th power $\mathfrak{J}^{n}$ of the ideal $\mathfrak{J}$ in $\mathcal{A}$ consists of all $(n+1) \times(n+1)$ matrices with zeroes in every entry with the possible exception of the upper right-hand corner, that is, the $(1 \ldots n)$-th entry. The ideal $\mathfrak{J}^{n}$ is also the center of $\mathfrak{J}$ with its Lie algebra structure. Moreover, the group $G$ acts trivially on $\mathfrak{J}^{n}$ via right and left multiplication as well as via the adjoint action. For if $Y \in \mathfrak{J}^{n}$ and $X \in \mathfrak{J}$, then $X Y=Y X=0$, since $\mathfrak{J}^{n+1}=\{0\}$. Thus

$$
Y(I+X)=Y=(I+X) Y
$$

and, using (2.1),

$$
(I+X)^{-1} Y(I+X)=Y .
$$

Furthermore, the $n$-th central series subgroup of $G$ is

$$
G^{(n)}=\left\{I+X: X \in \mathfrak{J}^{n}\right\} .
$$

Definition 2.1.1. A nilpotent connection $\omega$ whose curvature $\Omega$ lies in the ideal $\mathfrak{J}^{n}$ will be said to have central curvature.

In matrix form (2.5) this means that all entries $\Omega_{r \ldots s}=0$ except possibly for $\Omega_{12 \ldots n}$. Clearly, if $\omega$ has central curvature, then $\Omega \wedge \omega=\omega \wedge \Omega=0$, which implies $d \Omega=0$ by Bianchi's identity (2.6). In particular, $d \Omega_{12 \ldots n}=0$. Consequently $\Omega_{12 \ldots n}$ represents an element of the second de Rham cohomology group $H^{2}(M)$. In fact, the cohomology class $\left[\Omega_{12 \ldots n}\right]$ is an element of the Massey product $\left\langle\left[\omega_{1}\right], \ldots,\left[\omega_{n}\right]\right\rangle$ of the first degree cohomology classes represented by the 1 -forms, $\omega_{1}, \ldots, \omega_{n}$. Indeed, the structure equation (2.4) is exactly the set of equations defining the Massey product [5, p. 233]. Recall that a Massey product is a set of cohomology classes which may have more than one element because of indeterminancy.

Conversely, given an element of the Massey product $\left\langle\beta_{1}, \ldots, \beta_{n}\right\rangle$ of a set of cohomology classes, $\beta_{1}, \ldots, \beta_{n}$ in $H^{1}(M)$, take a defining set of 1 -forms $\omega_{r \ldots s}$ for the Massey product. Then equation (2.3) defines a nilpotent connection having central curvature for which $\Omega_{12 . . n}$ represents the given element of $\left\langle\beta_{1}, \ldots, \beta_{n}\right\rangle$ (cf. [11, §3]).

2.2. Holonomy. Let $\gamma:[0,1] \rightarrow M$ be a (piecewise) smooth curve in $M$. The holonomy, or parallel transport, along $\gamma$ is the element

$$
U(\gamma)=Y(1) \in G,
$$

where $Y(t)$ is the solution of the matrix differential equation

$$
Y^{\prime}(t)=Y(t) \omega(\gamma(t))
$$


satisfying $Y(0)=I$. There is an explicit formula for the holonomy expressed as a sum of iterated integrals due to Chen [4],

$$
U(\gamma)=I+\sum_{k=1}^{n} \int \cdots \int_{0 \leq t_{1} \leq \cdots \leq t_{k} \leq 1} \omega\left(\dot{\gamma}\left(t_{1}\right)\right) \cdots \omega\left(\dot{\gamma}\left(t_{k}\right)\right) d t_{1} \cdots d t_{k} .
$$

The general formula in [4 is an infinite series that arises from Picard iteration. In our case, equation (2.10) is a finite sum because $\mathfrak{J}$ is a nilpotent ideal.

Remark 2.2.1. In the viewpoint of the connection form $\hat{\omega}$ in the principal bundle $M \times G$,

$$
U(\gamma)=\hat{\gamma}(1)^{-1} \in G,
$$

where $(\gamma(t), \hat{\gamma}(t))$ is the $\hat{\omega}$-horizontal lift of $\gamma$ satisfying $\hat{\gamma}(0)=I$ [8, p. 68] since $\hat{\gamma}(t)=Y^{-1}(t)$.

Holonomy induces a group homomorphism $U: \mathcal{L}(*) \rightarrow G$, where $\mathcal{L}(*)$ is the loop group based at a point $* \in M$. The elements of $\mathcal{L}(*)$ are equivalence classes of loops based at $*$, and the group product is induced from the product of paths. Two loops are considered equivalent if they differ by a sequence of elementary "retracing" equivalences and oriented reparametrizations. Equivalent loops have the same holonomy. For more details, see [16, 3].

2.3. A Gauss-Bonnet formula for parallel transport. Suppose that $\gamma:[0,1]$ $\rightarrow M$ is a contractible loop in $M$. This means that there is a smooth homotopy of loops $\gamma_{s}:[0,1] \rightarrow M(0 \leq s \leq 1)$ between the curve $\gamma=\gamma_{1}$ and the constant path $\gamma_{0}$. We can regard the homotopy as a mapping $x:[0,1] \times[0,1] \rightarrow M$ by $x(t, s)=\gamma_{s}(t)$. As noted in the introduction the following is well known.

Theorem 2.3.1. Suppose $\omega$ is a nilpotent connection with curvature form $\Omega$. Let $\gamma:[0,1] \rightarrow M$ be a contractible loop in $M$, let $\gamma_{s}:[0,1] \rightarrow M$ be a smooth homotopy of loops in $M$ between the curve $\gamma=\gamma_{1}$ and the constant path $\gamma_{0}$, and let $\left(\gamma_{s}, \hat{\gamma}_{s}\right)$ denote the horizontal lift of $\gamma_{s}$ satisfying $\hat{\gamma}_{s}(0)=I(0 \leq s \leq 1)$. Then

$$
\hat{\gamma}(1)=I+\int_{0}^{1} \hat{\gamma}_{s}(1) \int_{0}^{1} \hat{\gamma}_{s}(t)^{-1} \Omega\left(x_{*}\left(\partial_{t}\right), x_{*}\left(\partial_{s}\right)\right) \hat{\gamma}_{s}(t) d t d s .
$$

A proof in more generality can be found in [13, Corollary 3]. One can also deduce it as a very special case of [2, Theorem 4.1]. Other versions of this theorem can be found in the literature, sometimes under the name, "The Non-abelian Stokes Theorem", for example, [17, 11. The earliest version is in [15.

Corollary 2.3.2. Assume $\omega$ is a nilpotent connection with central curvature $\Omega$. Let $\gamma:[0,1] \rightarrow M$ be a contractible loop in $M$, and let $x:[0,1] \times[0,1] \rightarrow M$ be a smooth homotopy of loops in $M$ between the curve $\gamma$ and the constant path. Then

$$
U(\gamma)=I-\int_{x} \Omega
$$

Proof. By assumption, the values of $\Omega$ lie in $\mathfrak{J}^{n}$. But $G$ acts trivially on $\mathfrak{J}^{n}$ via the adjoint action (2.8) and left multiplication (2.7). Thus, Theorem 2.3.1 simplifies to

$$
\hat{\gamma}(1)=I+\int_{0}^{1} \int_{0}^{1} \Omega\left(x_{*}\left(\partial_{t}\right), x_{*}\left(\partial_{s}\right)\right) d t d s
$$


Since the value of the double integral lies in $\mathfrak{J}^{n}$, its square and higher powers are zero. Therefore, by equation (2.11) and the inverse formula (2.1),

$$
U(\gamma)=\hat{\gamma}(1)^{-1}=I-\int_{0}^{1} \int_{0}^{1} \Omega\left(x_{*}\left(\partial_{t}\right), x_{*}\left(\partial_{s}\right)\right) d t d s .
$$

2.4. Quotient connections. We apply well-known properties of mappings of connections [8, pp. 79-82] to obtain information about the holonomy of $\omega$ by means of certain quotient connections $\bar{\omega}$.

Let $\mathfrak{J}_{0}$ be a two-sided ideal of $\mathcal{A}$ with $\mathfrak{J}_{0} \subset \mathfrak{J}$. Then $\mathfrak{J}_{0}$ is a Lie algebra ideal of $\mathfrak{J}$, and

$$
G_{0}=\left\{I+X: X \in \mathfrak{J}_{0}\right\}
$$

is a closed normal Lie subgroup of $G$ whose Lie algebra is $\mathfrak{J}_{0}$. Let

$$
\Phi: A \rightarrow \overline{\mathcal{A}}=\mathcal{A} / \mathfrak{J}_{0}, \quad \phi_{*}: \mathfrak{J} \rightarrow \overline{\mathfrak{J}}=\mathfrak{J} / \mathfrak{J}_{0}, \quad \text { and } \quad \phi: G \rightarrow \bar{G}=G / G_{0}
$$

denote the respective algebra, Lie algebra, and group quotient homomorphisms. Obviously, $\overline{\mathfrak{J}}$ is the Lie algebra of $\bar{G}$, and $\phi_{*}=\Phi \mid \mathfrak{J}$ is the Lie algebra homomorphism induced from the Lie group homomorphism $\phi$.

Proposition 2.4.1. Let $\omega$ be a $\mathfrak{J}$-valued 1 -form on $M$. The $\overline{\mathfrak{J}}$-valued 1 -form $\bar{\omega}=\phi_{*} \omega$ defines a connection in $M \times \bar{G}$ whose curvature satisfies $\bar{\Omega}=\phi_{*} \Omega$ and whose holonomy satisfies $\bar{U}(\gamma)=\phi(U(\gamma))$ for every curve $\gamma$ in $M$.

In case $\omega$ has central curvature and $\mathfrak{J}^{n} \subset \mathfrak{J}_{0}$, then $\bar{\omega}$ is flat and $\phi(U(\gamma))$ depends only on the homotopy class of $\gamma$.

Proof. Apply Proposition 6.1 of [8, pp. 79-80] to the bundle homomorphism $i d \times \phi$ : $M \times G \rightarrow M \times \bar{G}$ and the connection $\hat{\omega}$ in $M \times G$ associated to $\omega$ to obtain the unique connection $\hat{\bar{\omega}}$ in $M \times \bar{G}$ that satisfies $\phi_{*} \hat{\omega}=(i d \times \phi)^{*} \hat{\bar{\omega}}, \phi_{*} \hat{\Omega}=(i d \times \phi)^{*} \hat{\bar{\Omega}}$, and such that $i d \times \phi$ carries $\hat{\omega}$-horizontal curves to $\hat{\bar{\omega}}$-horizontal curves. If $\bar{\sigma}=(i d \times \phi) \circ \sigma$ : $M \rightarrow M \times \bar{G}$ is the canonical section, then $\bar{\sigma}^{*} \hat{\bar{\omega}}=\phi_{*}(\omega)=\bar{\omega}, \bar{\Omega}=\bar{\sigma}^{*} \hat{\bar{\Omega}}=\phi_{*} \Omega$, and $\bar{U}(\gamma)=\phi(U(\gamma))$ for every curve $\gamma$ in $M$.

Now assume $\omega$ has central curvature and $\mathfrak{J}^{n} \subset \mathfrak{J}_{0}$. Thus $\phi_{*}\left(\mathfrak{J}^{n}\right)=\{0\}$ in $\overline{\mathfrak{J}}$. Therefore $\bar{\Omega}=\phi_{*} \Omega=0$. Since the holonomy of a curve in a flat connection depends only on the homotopy class of the curve, $\phi(U(\gamma))=\bar{U}(\gamma)$ depends only on the homotopy class of $\gamma$.

By a similar use of the methods of [8, pp. 79-82], we can prove the following.

Proposition 2.4.2. Suppose $f: M \rightarrow \bar{M}$ is a smooth map. If $\omega$ is a $\mathfrak{J}$-valued $1-$ form on $M$ and $\bar{\omega}$ is a $\overline{\mathfrak{J}}-$ valued 1 -form on $\bar{M}$ such that $f^{*} \bar{\omega}=\phi_{*} \omega$, then $f^{*} \bar{\Omega}=\phi_{*} \Omega$ and $\bar{U}(f \circ \gamma)=\phi(U(\gamma))$ for every curve $\gamma$ in $M$.

Corollary 2.4.3. In addition, suppose that $f: M \rightarrow \bar{M}$ is an open dense embedding of $M$ in $\bar{M}$, that $\omega$ has central curvature, and that $\mathfrak{J}^{n} \subset \mathfrak{J}_{0}$. If $\gamma$ is a loop in $M$ such that $f \circ \gamma$ is contractible in $\bar{M}$, then $U(\gamma) \in G_{0}$.

Proof. By Proposition 2.4.2 the curvature assumptions imply that $f^{*} \bar{\Omega}=\phi_{*} \Omega=0$. Hence $\bar{\Omega}=0$ on $\bar{M}$ because $f$ is an open dense embedding. Thus the contractibility of $f \circ \gamma$ in $\bar{M}$ implies $\phi(U(\gamma))=\bar{U}(f \circ \gamma)=I$ in $\bar{G}$. Therefore $U(\gamma) \in \operatorname{ker} \phi=G_{0}$. 
Remark 2.4.4. Except for the statements specific to upper triangular matrices and Massey products, all the definitions and results of $\S 2$ are valid more generally for any finite dimensional, real associative algebra $\mathcal{A}$ with identity possessing a 2 -sided nilpotent ideal $\mathfrak{J}$ satisfying $\mathfrak{J}^{n+1}=\{0\}$.

\section{LINK INVARIANTS}

From now on let $M$ be the complement of an oriented link $L=L_{1} \cup \cdots \cup L_{N}$ of $N$-components in $S^{3}$. Let $T_{i}$ be the toral boundary of a tubular neighborhood of the $i$-th link component $L_{i}$. If $S^{3}$ is given its usual orientation, we orient $T_{i}$ by the inward normal vector field.

A longitude $l_{i}$ in $T_{i}$ is a simple oriented loop having linking number 0 with $L_{i}$ and which is homotopic to $L_{i}$ in the tubular neighborhood, while a meridian $m_{i}$ in $T_{i}$ is a simple oriented loop in $T_{i}$ intersecting $l_{i}$ in exactly one point, having linking number +1 with $L_{i}$ and which is homotopically trivial in the tubular neighborhood. The commutator $\left[m_{i}, l_{i}\right]$ is contractible in $T_{i}$. A contracting homotopy $x:[0,1] \times[0,1] \rightarrow$ $T_{i}$ can be constructed as follows. Cut $T_{i}$ along $m_{i}$ and $l_{i}$ to obtain a rectangle $R$ whose boundary is the loop $m_{i} l_{i} m_{i}^{-1} l_{i}^{-1}$ based at its lower left-hand corner $c$. Define $x:[0,1] \times[0,1] \rightarrow R$ to be a homotopy in $R$ from $x(-, 1)=m_{i} l_{i} m_{i}^{-1} l_{i}^{-1}$ to the constant path $x(-, 0)=c$ which restricts to a diffeomorphism between the interiors of $[0,1] \times[0,1]$ and $R$. Note that $x$ reverses orientation. Therefore, for every 2 -form $\Omega$ in $M$,

$$
\int_{x} \Omega=-\int_{T_{i}} \Omega
$$

We will require the set of meridians and longitudes to be based at a fixed point $* \in M$. For each $i$, choose a path $p_{i}$ from $*$ to the point of intersection of $m_{i}$ and $l_{i}$ in $T_{i}$, and replace $m_{i}$ and $l_{i}$ respectively by $p_{i} m_{i} p_{i}^{-1}$ and $p_{i} l_{i} p_{i}^{-1}$. Renaming these new loops $m_{i}$ and $l_{i}$ as before, the new commutator $\left[m_{i}, l_{i}\right]$ is trivial in the fundamental group $\pi_{1}$ of $M$ based at $*$. By constructing its contracting homotopy $x$ in two stages, first contracting $\left[m_{i}, l_{i}\right]$ to $p_{i} p_{i}^{-1}$, using the previous homotopy in $T_{i}$, and then contracting $p_{i} p_{i}^{-1}$ back to $*$ along $p_{i}$, the identity (3.1) continues to hold.

3.1. Milnor invariants. Let $\pi_{1}^{(n)}$ denote the $n$-th lower central series subgroup of $\pi_{1}$. It is known that the meridians $m_{1}, \ldots, m_{N}$ generate the factor group $\pi_{1} / \pi_{1}^{(n)}$. Thus the image of each longitude $l_{i}$ in $\pi_{1} / \pi_{1}^{(n)}$ can be expressed as a word $w_{i}$ in the meridians. The Milnor invariants are defined by means of the Magnus expansions of the $w_{i}$.

Let $F$ denote the free group on the letters $m_{1}, \ldots, m_{N}$, let $\mathcal{P}$ denote the associative algebra of formal power series in the noncommuting indeterminates $x_{1}, \ldots, x_{N}$, and let $\mathcal{P}^{1}$ denote the multiplicative group of power series in $\mathcal{P}$ whose constant term is 1 . The Magnus expansion is the group homomorphism

$$
\delta: F \rightarrow \mathcal{P}^{1}
$$

for which $\delta\left(m_{i}\right)=1+x_{i}$.

The coefficients in the Magnus expansion of the word $w_{i}$ define the Milnor numbers $\mu\left(i_{1}, \ldots, i_{k}, i\right)$, that is,

$$
\delta\left(w_{i}\right)=1+\sum \mu\left(i_{1}, \ldots, i_{k}, i\right) x_{i_{1}} \cdots x_{i_{k}} .
$$


For a given multi-index $\left(i_{1}, \ldots, i_{k}\right)$, it is known that the integer $\mu\left(i_{1}, \ldots, i_{k}\right)$ depends on the choices of the meridians and the longitudes. However, $\bar{\mu}\left(i_{1}, \ldots, i_{k}\right)$, which is defined to be its residue class modulo $\Delta\left(i_{1}, \ldots, i_{k}\right)$, does not depend on these choices. Here $\Delta\left(i_{1}, \ldots, i_{k}\right)$ is defined to be the greatest common divisor of the $\mu\left(j_{1}, \ldots, j_{r}\right)$, where $j_{1}, \ldots, j_{r}$ with $2 \leq r<k$ ranges over all sequences obtained from $i_{1}, \ldots, i_{k}$ by deleting at least one of the indices and permuting the remaining indices cyclically.

For more details on this section, see, for example, [5, pp. 211-224].

3.2. Massey numbers. The first cohomology group $H^{1}(M)$ has a basis $\alpha_{1}, \ldots, \alpha_{N}$ consisting of the Alexander duals of the corresponding link components $L_{1}, \ldots, L_{N}$. Given a multi-index $\left(i_{1}, \ldots, i_{n}\right)$, which may have repeated indices, assume that the associated Massey product $\left\langle\alpha_{i_{1}}, \ldots, \alpha_{i_{n}}\right\rangle$ is defined,. By Section 2.1 there is a nilpotent connection $\omega$ with central curvature $\Omega$ satisfying $\left[\omega_{k}\right]=\alpha_{i_{k}}(k=1, \ldots, n)$ such that $\left[\Omega_{1 \ldots n}\right]$ is an element of $\left\langle\alpha_{i_{1}}, \ldots, \alpha_{i_{n}}\right\rangle$. It is known that the tori $T_{1}, \ldots, T_{N}$ represent homology classes that span $H_{2}(M)$. Thus the cohomology class $\left[\Omega_{1 \ldots n}\right]$ is completely determined by the Massey numbers

$$
\int_{T_{i}} \Omega_{1 \ldots n}, \quad i=1, \ldots, N
$$

In particular, if all these integrals are zero, then $\left[\Omega_{1 \ldots n}\right]=0$ in $H^{2}(M)$.

Proposition 3.2.1. If $\omega$ is a connection associated to the Massey product $\left\langle\alpha_{i_{1}}, \ldots\right.$, $\left.\alpha_{i_{n}}\right\rangle$ as above, then the holonomy of the meridian $m_{j}$ takes the form

$$
U\left(m_{j}\right)=I+\sum_{k=1}^{n} \delta_{i_{k}}^{j} E^{k}+X_{j}
$$

where $X_{j} \in \mathfrak{J}^{2}$. The matrices $E^{k}$ were defined in $§ 2.1$.

Proof. Because $\left[\omega_{k}\right]$ is the Alexander dual of $L_{i_{k}}, \int_{m_{j}} \omega_{k}=\delta_{i_{k}}^{j}$, where $\delta_{i}^{j}$ is the Kronecker delta. The result follows by applying the iterated integral formula for holonomy (2.10).

3.3. Relations between the Massey and Milnor invariants. A basic relation holds among the Massey numbers, the Milnor numbers and the holonomy of the meridians.

Theorem 3.3.1. Assume $\omega$ is a nilpotent connection on $M$ with central curvature. Set $U\left(m_{i}\right)=I+K_{i}, K_{i} \in \mathfrak{J}$, for each $i \in\{1, \ldots, N\}$. Then for all $i=1, \ldots, N$,

$$
\int_{T_{i}} \Omega=\sum \mu\left(j_{1}, \ldots, j_{r}, i\right)\left[K_{i}, K_{j_{1}} \cdots K_{j_{r}}\right],
$$

where the sum extends over all multi-indices $\left(j_{1}, \ldots, j_{r}\right)$ with $r<n$.

Proof. Regard the meridians $m_{1}, \ldots, m_{N}$ and the longitudes $l_{1}, \ldots, l_{N}$ as elements of $\mathcal{L}(*)$. Passage to homotopy classes defines a group homomorphism $\mathcal{L}(*) \rightarrow \pi_{1}$, where $\pi_{1}$ is the fundamental group of $M$. The assignment $m_{i} \mapsto m_{i}$ extends to a group homomorphism from the free group $F$ on $m_{1}, \ldots, m_{N}$ to $\mathcal{L}(*)$. The words $w_{1}, \ldots, w_{N}$ are elements in $F$ which are equal to $l_{1}, \ldots, l_{N}$ respectively when mapped to the quotient group $\pi_{1} / \pi_{1}^{(n)}$.

Because $\mathfrak{J}^{n+1}=\{0\}$, the substitution of the indeterminates $x_{i}$ by the correspond$\operatorname{ing} K_{i} \in \mathfrak{J}$ reduces any given power series in $\mathcal{P}$ to a finite sum in $\mathcal{A}$. Consequently, 
this substitution defines an algebra homomorphism $s: \mathcal{P} \rightarrow \mathcal{A}$ that carries 1 to $I$. Restricting $s$ to $\mathcal{P}^{1}$ gives a group homomorphism $s: \mathcal{P}^{1} \rightarrow G$ for which $s\left(\delta\left(m_{i}\right)\right)=$ $I+K_{i}=U\left(m_{i}\right)$. Therefore, by (3.2), $s\left(\delta\left(w_{i}\right)\right)=I+\sum \mu\left(j_{1}, \ldots, j_{r}, i\right) K_{j_{1}} \cdots K_{j_{r}}$, where the sum extends over all $\left(j_{1}, \ldots, j_{r}\right)$ with $r \leq n$.

Let $\bar{\omega}$ be the flat quotient connection of $\omega$ obtained from Proposition 2.4.1 using the ideal $\mathfrak{J}_{0}=\mathfrak{J}^{n}$. The holonomy of $\bar{\omega}$ induces a group homomorphism $\bar{U}: \pi_{1} \rightarrow \bar{G}$, where $\bar{G}$ is the quotient of $G$ by $G^{(n)}=\left\{I+X: X \in \mathfrak{J}^{n}\right\}$ which is just the $n$-th lower central series subgroup of $G$. Thus $\bar{U}\left(\pi_{1}^{(n)}\right)$ is trivial and $\bar{U}$ factors through $\pi_{1} / \pi_{1}^{(n)}$.

The following commutative diagram summarizes this discussion:

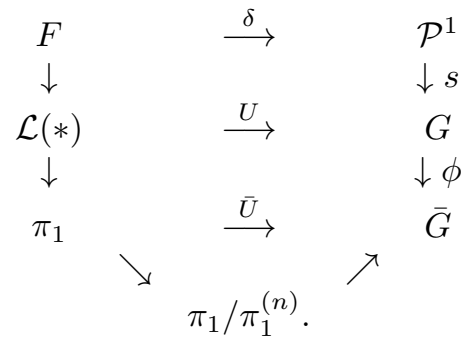

Since $l_{i}=w_{i}$ in $\pi_{1} / \pi_{1}^{(n)}, \bar{U}\left(l_{i}\right)=\bar{U}\left(w_{i}\right)=\phi\left(s\left(\delta\left(w_{i}\right)\right)\right)$ in $\bar{G}$. Thus

$$
U\left(l_{i}\right)=I+\sum \mu\left(j_{1}, \ldots, j_{r}, i\right) K_{j_{1}} \cdots K_{j_{r}}+Z_{i}
$$

where $Z_{i} \in \mathfrak{J}^{n}$ and the sum extends over all $\left(j_{1}, \ldots, j_{r}\right)$ with $r \leq n$. Let

$$
\Lambda_{i}=\sum \mu\left(j_{1}, \ldots, j_{r}, i\right) K_{j_{1}} \cdots K_{j_{r}}+Z_{i}
$$

Then, since $\mathfrak{J}^{n+1}=\{0\}$,

$$
\left[K_{i}, \Lambda_{i}\right]=\sum \mu\left(j_{1}, \ldots, j_{r}, i\right)\left[K_{i}, K_{j_{1}} \cdots K_{j_{r}}\right]
$$

where the sum extends over all $\left(j_{1}, \ldots, j_{r}\right)$ with $r<n$. On the other hand, applying Corollary 2.3.2 with $\gamma=\left[m_{i}, l_{i}\right]$, together with equation (3.1), shows

$$
U\left(m_{i}\right) U\left(l_{i}\right) U\left(m_{i}\right)^{-1} U\left(l_{i}\right)^{-1}-I=U\left(\left[m_{i}, l_{i}\right]\right)-I=\int_{T_{i}} \Omega \in \mathfrak{J}^{n} .
$$

Now the Lie bracket $\left[K_{i}, \Lambda_{i}\right]$ can be calculated in another way. Using (3.4) and the triviality of the right action (2.7) of $G$ on $\mathfrak{J}^{n}$,

$$
\begin{aligned}
{\left[K_{i}, \Lambda_{i}\right] } & =\left(I+K_{i}\right)\left(I+\Lambda_{i}\right)-\left(I+\Lambda_{i}\right)\left(I+K_{i}\right) \\
& =U\left(m_{i}\right) U\left(l_{i}\right)-U\left(l_{i}\right) U\left(m_{i}\right) \\
& =\left(U\left(\left[m_{i}, l_{i}\right]\right)-I\right) U\left(l_{i}\right) U\left(m_{i}\right) \\
& =\int_{T_{i}} \Omega .
\end{aligned}
$$

Equating these two formulas for $\left[K_{i}, \Lambda_{i}\right]$ completes the proof. 
Corollary 3.3.2. Let $n \geq 2$. If $\mu\left(j_{1}, \ldots, j_{s}\right)=0$ for all $s<n$, then for every multiindex $\left(i_{1}, \ldots, i_{n}\right)$ of length $n$, the Massey product $\left\langle\alpha_{i_{1}}, \ldots, \alpha_{i_{n}}\right\rangle$ is defined, and for every nilpotent connection $\omega$ with central curvature $\Omega$ associated to $\left\langle\alpha_{i_{1}}, \ldots, \alpha_{i_{n}}\right\rangle$,

$$
\int_{T_{i}} \Omega_{1 \ldots n}=\left\{\begin{array}{cl}
\mu\left(i_{2}, \ldots, i_{n}, i_{1}\right) & \text { if } i=i_{1} \neq i_{n} \\
-\mu\left(i_{1}, \ldots, i_{n}\right) & \text { if } i=i_{n} \neq i_{1} \\
0 & \text { otherwise }
\end{array}\right.
$$

for all $i=1, \ldots, N$.

Proof. This is proved by induction on $n$. When $n=2$, the Massey product is just the cup product $\alpha_{i_{1}} \cup \alpha_{i_{2}}$, and both sides of (3.5) equal the linking number of $L_{i_{1}}$ with $L_{i_{2}}$.

Having established the result for $n$ from 2 through $m-1$, assume all Milnor numbers of order $<m$ vanish. By the inductive hypothesis, all Massey products of order $<m$ vanish because by (3.5) these products evaluate to zero on all the $T_{i}$, since the relevant Milnor numbers on the right-hand side of (3.5) are assumed to vanish. Thus the $m$-th order Massey products are defined. In particular, given $\left(i_{1}, \ldots, i_{m}\right)$, there is a connection $\omega$ with central curvature associated to the Massey product $\left\langle\alpha_{i_{1}}, \ldots, \alpha_{i_{m}}\right\rangle$. Since all Milnor numbers of order $<m$ vanish, the sum in Theorem 3.3.1 contains only the $m$-th order terms:

$$
\begin{aligned}
\int_{T_{i}} \Omega & =\sum \mu\left(j_{1}, \ldots, j_{m-1}, i\right)\left[K_{i}, K_{j_{1}} \cdots K_{j_{m-1}}\right] \\
& =\sum \mu\left(j_{1}, \ldots, j_{m-1}, i\right)\left(K_{i} K_{j_{1}} \cdots K_{j_{m-1}}-K_{j_{1}} \cdots K_{j_{m-1}} K_{i}\right)
\end{aligned}
$$

summed over all $\left(j_{1}, \ldots, j_{m-1}\right)$. By Proposition 3.2.1, recalling that $U\left(m_{j}\right)=$ $I+K_{j}$,

$$
K_{j}=\sum_{k=1}^{m} \delta_{i_{k}}^{j} E^{k}+X_{j}
$$

where $X_{j} \in \mathfrak{J}^{2}$. By repeated applications of equation (2.2), using $\mathfrak{J}^{m+1}=\{0\}$, one finds that every $m$-fold product of the $K_{j}$ 's is zero except

$$
K_{i_{1}} \cdots K_{i_{m}}=E^{1 \ldots m} .
$$

Therefore every term in the sum (3.6) vanishes, except when either $\left(i, j_{1}, \ldots, j_{m-1}\right)$ or $\left(j_{1}, \ldots, j_{m-1}, i\right)$ is equal to $\left(i_{1}, \ldots, i_{m}\right)$. Examining these two exceptions, case by case, leads to the equation:

$$
\int_{T_{i}} \Omega_{1 \ldots m}=\left\{\begin{array}{cl}
\mu\left(i_{2}, \ldots, i_{m}, i_{1}\right) & \text { if } i=i_{1} \neq i_{m} \\
-\mu\left(i_{1}, \ldots, i_{m}\right) & \text { if } i=i_{m} \neq i_{1} \\
\mu\left(i_{2}, \ldots, i_{m}, i_{1}\right)-\mu\left(i_{1}, \ldots, i_{m}\right) & \text { if } i=i_{1}=i_{m} \\
0 & \text { otherwise }
\end{array}\right.
$$

From (3.7) it immediately follows that

$$
\sum_{i=1}^{N} \int_{T_{i}} \Omega_{1 \ldots m}=\mu\left(i_{2}, \ldots, i_{m}, i_{1}\right)-\mu\left(i_{1}, \ldots, i_{m}\right)
$$

whether or not $i_{1}=i_{m}$. Consequently, applying Stokes Theorem, we obtain

$$
\mu\left(i_{2}, \ldots, i_{m}, i_{1}\right)-\mu\left(i_{1}, \ldots, i_{m}\right)=\sum_{i=1}^{N} \int_{T_{i}} \Omega_{1 \ldots m}=\int_{\partial M} \Omega_{1 \ldots m}=\int_{M} d \Omega_{1 \ldots m}=0
$$


since $d \Omega_{1 \ldots m}=0$ because of central curvature. This completes the induction step and the proof of the corollary.

Remark 3.3.3. (1) The statement of Corollary 3.3 .2 remains true if the $\mu$ 's are replaced by the corresponding $\bar{\mu}$ 's. This is because if all the Milnor numbers of order $<n$ vanish, whether the $\mu$ 's or the $\bar{\mu}$ 's, then each $\mu$ of order $\leq n$ equals the corresponding $\bar{\mu}$.

(2) The fact that $\mu\left(i_{2}, \ldots, i_{m}, i_{1}\right)-\mu\left(i_{1}, \ldots, i_{m}\right)=0$ in the proof of Corollary 3.3.2 demonstrates a special case of Milnor's result about the invariance of the $\bar{\mu}\left(j_{1}, \ldots, j_{s}\right)$ under a cyclic permutation of the indices $\left(j_{1}, \ldots, j_{s}\right)$.

Corollary 3.3.4. Let $n \geq 3$. If all Massey products of order $<n$ vanish, then the conclusion of Corollary 3.3.2, in particular, equation (3.5), holds.

Proof. We first observe that the conclusion of Corollary 3.3.2 is still true under the weaker assumption that $\mu\left(j_{1}, \ldots, j_{s}\right)=0$ for all $s<n$ whenever the $j$ 's are not all the same. The induction argument in Corollary 3.3 .2 will still work provided equation (3.6) remains valid under the weaker assumptions. Indeed, all the terms of order $<n$ in the sum of Theorem 3.3.1 must vanish because, if $\left(j_{1}, \ldots, j_{r}, i\right)$ are all the same, then the Lie bracket $\left[K_{i}, K_{j_{1}} \cdots K_{j_{r}}\right]$ is zero, and if not, then the coefficient $\mu\left(j_{1}, \ldots, j_{r}, i\right)$ is zero.

Thus it suffices to prove that if all Massey products of order less than $n$ vanish, then $\mu\left(j_{1}, \ldots, j_{s}\right)=0$ for all $s<n$ whenever the $j$ 's are not all the same. For fixed $n \geq 3$ we will prove this by induction over $s$. The induction starts with $s=2$. Indeed the hypothesis implies that all order 2 linking numbers vanish.

Next assume that $r<n$ and that we have proved that $\mu\left(j_{1}, \ldots, j_{s}\right)=0$ for all $s<r$ whenever the $j$ 's are not all the same. Take a multi-index $\left(i_{1}, \ldots, i_{r}\right)$ where the $i$ 's are not all the same. We proceed to show $\mu\left(i_{1}, \ldots, i_{r}\right)=0$. By assumption $\left\langle\alpha_{i_{1}}, \ldots, \alpha_{i_{r}}\right\rangle=0$. Let $\omega$ be a connection with central curvature associated to $\left\langle\alpha_{i_{1}}, \ldots, \alpha_{i_{r}}\right\rangle$. The inductive assumption and the above observation imply that (3.5) holds. In case $i_{1} \neq i_{r}$, equation (3.5) implies

$$
0=\int_{T_{i_{r}}} \Omega_{1 \ldots r}=-\mu\left(i_{1}, \ldots, i_{r}\right),
$$

which gives the desired result: $\mu\left(i_{1}, \ldots, i_{r}\right)=0$. However, if $i_{1}=i_{r}$, we may cyclically permute the indices so that the first index does not equal the last. The result follows from the first case by the previous remark.

Corollaries 3.3 .2 and 3.3 .4 should be compared to the versions of the PorterTuraev Theorem in [18, [12, Theorem 3], and [5, Theorem 6.4.3].

3.4. Reducible connections. A restricted type of Massey product is employed in [12. For a given multi-index $\left(i_{1}, \ldots, i_{n}\right)$, it is called a Massey product in the system $\left\{S^{3} \backslash L_{i_{k}}\right\}_{k=1}^{n}$. We incorporate this notion into our approach through the definition of a reducible connection. We call such connections reducible because they induce quotient connections for sublinks.

Definition 3.4.1. A nilpotent connection (2.3) on $M$ is reducible with respect to $\left(i_{1}, \ldots, i_{n}\right)$ if for every $1 \leq r \leq s \leq n$, the 1 -form $\omega_{r \ldots s}$ extends to a smooth 1 -form on $S^{3} \backslash\left(L_{i_{r}} \cup \cdots \cup L_{i_{s}}\right)$. 
Our next theorem relates an $n$-th order Massey product to the corresponding $\bar{\mu}$ even if not all the lower order $\mu$ 's vanish. It can be regarded as a weak version of [12, Theorem 3]. In order to state it, one more definition is needed.

Definition 3.4.2. A nilpotent connection with central curvature is said to have integral holonomy if for every loop $\gamma$, every entry of the holonomy matrix $K=$ $U(\gamma)-I$ is an integer, with the one possible exception of $K_{1 \ldots n}$. Clearly, any product of two or more such holonomy matrices has only integer entries, with no exceptions.

Theorem 3.4.3. If $\omega$ is a connection, reducible with respect to $\left(i_{1}, \ldots, i_{n}\right)$, with central curvature $\Omega$ associated $\left\langle\alpha_{i_{1}}, \ldots, \alpha_{i_{n}}\right\rangle$, and having integral holonomy, then

$$
\int_{T_{i}} \Omega_{1 \ldots n} \equiv\left\{\begin{array}{cl}
\mu\left(i_{2}, \ldots, i_{n}, i_{1}\right) & \text { if } i=i_{1} \neq i_{n} \\
-\mu\left(i_{1}, \ldots, i_{n}\right) & \text { if } i=i_{n} \neq i_{1} \\
0 & \text { otherwise. }
\end{array}\right.
$$

where the congruence $\equiv$ is taken $\bmod \Delta\left(i_{1}, \ldots, i_{n}\right)$.

Proof. Set $U\left(m_{j}\right)=I+K_{j}, K_{j} \in \mathfrak{J}$, for each $j=1, \ldots, N$. The next two lemmas allow us to simplify the right-hand side of the equation in Theorem 3.3 .1 .

Lemma 3.4.4. Let $j \in\{1, \ldots, N\}$. If $j$ is one of the indices in $\left(i_{1}, \ldots, i_{n}\right)$, then $K_{j}$ is a linear combination of the basis matrices $E^{r \ldots s}$ for which there exists some $k$ satisfying $i_{k}=j$ and $r \leq k \leq s$. If not, $K_{j}$ is a multiple of $E^{1 \ldots n}$.

Proof. Fix $j \in\{1, \ldots, N\}$. We first construct a suitable flat quotient connection. In case $j$ is one of the $\left(i_{1}, \ldots, i_{n}\right)$, define $\mathfrak{J}_{0}$ to be the vector subspace of $\mathfrak{J}$ spanned by the $E^{r \ldots s}$ such that for some $k, i_{k}=j$ and $r \leq k \leq s$. Otherwise set $\mathfrak{J}_{0}=\mathfrak{J}^{n}$. In either case, $E^{1 \ldots n} \in \mathfrak{J}_{0}$. Therefore, $\mathfrak{J}_{0}$ is clearly a 2 -sided ideal in $\mathcal{A}$ such that $\mathfrak{J}^{n} \subset \mathfrak{J}_{0}$.

Set $\bar{M}=M \cup L_{j}$, and let $f: M \rightarrow \bar{M}$ be the inclusion. Then $f$ is an open dense embedding, and $f \circ m_{j}$ is contractible in $\bar{M}$.

Using the notation of $\S 2.4$, let $\phi_{*}: \mathfrak{J} \rightarrow \mathfrak{J} / \mathfrak{J}_{0}$ denote the quotient Lie algebra homomorphism, and write

$$
\omega=\sum_{r \ldots s} \omega_{r \ldots s} E^{r \ldots s} .
$$

We will show that the quotient connection

$$
\phi_{*} \omega=\sum_{r \ldots s} \omega_{r \ldots s} \phi_{*}\left(E^{r \ldots s}\right)
$$

on $M$ extends to a smooth connection $\bar{\omega}$ on $\bar{M}$ such that $\phi_{*} \omega=f^{*} \bar{\omega}$. To do this it suffices to show that if $\phi_{*}\left(E^{r \ldots s}\right) \neq 0$ in $\mathfrak{J} / \mathfrak{J}_{0}$, then $w_{r \ldots s}$ extends smoothly to $\bar{M}$. But, if $\phi_{*}\left(E^{r \ldots s}\right) \neq 0$, then $E^{r \ldots s} \notin \mathfrak{J}_{0}$. Hence there is no $k$ such that $r \leq k \leq s$ with $i_{k}=j$. Thus $L_{j} \subset S^{3} \backslash\left(L_{i_{r}} \cup \cdots \cup L_{i_{s}}\right)$. The reducibility of $\omega$ implies that $\omega_{r \ldots s}$ extends smoothly to $S^{3} \backslash\left(L_{i_{r}} \cup \cdots \cup L_{i_{s}}\right) \supset \bar{M}$. Thus $\phi_{*} \omega$ extends smoothly to $\bar{M}$, as required.

Having verified the hypothesis of Corollary 2.4.3 with $\gamma=m_{j}$, we conclude $U\left(m_{j}\right)=I+K_{j}$, where $K_{j} \in \mathfrak{J}_{0}$. This completes the proof of the lemma by definition of $\mathfrak{J}_{0}$. 
Lemma 3.4.5. Given $\left(j_{1}, \ldots, j_{m}\right), m \geq 2$, the $m$-fold product

$$
K_{j_{1}} \cdots K_{j_{m}}=0
$$

unless $\left(j_{1}, \ldots, j_{m}\right)$ is a subsequence of $\left(i_{1}, \ldots, i_{n}\right)$. Moreover, the only nonzero $n$-fold product is

$$
K_{i_{1}} \cdots K_{i_{n}}=E^{1 \ldots n} .
$$

Proof. If for some $\ell \in\{1, \ldots, m\}, K_{j_{\ell}}$ is a multiple of $E^{1 \ldots n}$, then $K_{j_{1}} \cdots K_{j_{m}}=0$ because $m \geq 2$ and $\mathfrak{J}^{n+1}=\{0\}$. Thus, by Lemma 3.4.4. we may assume that each $K_{j_{\ell}}$ is a linear combination of $E^{r_{\ell} \ldots s_{\ell}}$ for which there exist $k_{\ell}$ satisfying

$$
i_{k_{\ell}}=j_{\ell} \quad \text { and } \quad r_{\ell} \leq k_{\ell} \leq s_{\ell} .
$$

Thus the product $K_{j_{1}} \cdots K_{j_{m}}$ expands as a linear combination of the product expressions

$$
E^{r_{1} \ldots s_{1}} E^{r_{2} \ldots s_{2}} \cdots E^{r_{m} \ldots s_{m}}
$$

that satisfy (3.8). By equation (2.2), such products (3.9) vanish unless

$$
s_{1}+1=r_{2}, \quad s_{2}+1=r_{3}, \quad \ldots \quad, s_{m-1}+1=r_{m} .
$$

If (3.9) does not vanish, (3.8) and (3.10) imply that $k_{1}<k_{2}<\cdots<k_{m}$ and that $\left(j_{1}, \ldots, j_{m}\right)=\left(i_{k_{1}}, \ldots, i_{k_{m}}\right)$ is a subsequence of $\left(i_{1}, \ldots, i_{n}\right)$. Moreover, taking $m=n$, the only nonzero $n$-fold product is $K_{i_{1}} \cdots K_{i_{n}}$, which evaluates to $E^{1 \ldots n}$ on account of Proposition 3.2.1 $\mathfrak{J}^{n+1}=\{0\}$, and equation (2.2).

Coming back to the proof of Theorem 3.4.3, by Theorem 3.3.1,

$$
\int_{T_{i}} \Omega=\sum \mu\left(j_{1}, \ldots, j_{r}, i\right)\left[K_{i}, K_{j_{1}} \cdots K_{j_{r}}\right],
$$

where the sum extends over all multi-indices $\left(j_{1}, \ldots, j_{r}\right)$ with $r<n$. First consider the terms with $1 \leq r<n-1$. By Lemma 3.4.5. $\left[K_{i}, K_{j_{1}} \cdots K_{j_{r}}\right]=0$, unless either $\left(i, j_{1}, \ldots, j_{r}\right)$ or $\left(j_{1}, \ldots, j_{r}, i\right)$ is a proper subsequence of $\left(i_{1}, \ldots, i_{n}\right)$. Thus the coefficients $\mu\left(j_{1}, \ldots, j_{r}, i\right)$ corresponding to the nonvanishing brackets are divisible by $\Delta\left(i_{1}, \ldots, i_{n}\right)$. (See $\S 3.1$.) Also, because $\omega$ has integral holonomy, all the entries of $\left[K_{i}, K_{j_{1}} \cdots K_{j_{r}}\right]$ are integers. Therefore, working in the integers modulo $\Delta\left(i_{1}, \ldots, i_{n}\right)$, all the terms of (3.11) with $r<n-1$ vanish. Next consider the terms with $r=n-1$. Again by Lemma 3.4.5. $\left[K_{i}, K_{j_{1}} \cdots K_{j_{n-1}}\right]=0$ unless either $\left(i, j_{1}, \ldots, j_{n-1}\right)$ or $\left(j_{1}, \ldots, j_{n-1}, i\right)$ is equal to $\left(i_{1}, \ldots, i_{n}\right)$. Since $K_{i_{1}} \cdots K_{i_{n}}=$ $E^{1 \ldots n}$, a case-by-case inspection of the $(1 \ldots n)$-th entry of both sides of (3.11), similar to that in the proof of Corollary 3.3.2, leads to the following congruence modulo $\Delta\left(i_{1}, \ldots, i_{n}\right)$ :

$$
\int_{T_{i}} \Omega_{1 \ldots n} \equiv\left\{\begin{array}{cl}
\mu\left(i_{2}, \ldots, i_{n}, i_{1}\right) & \text { if } i=i_{1} \neq i_{n} \\
-\mu\left(i_{1}, \ldots, i_{n}\right) & \text { if } i=i_{n} \neq i_{1} \\
\mu\left(i_{2}, \ldots, i_{n}, i_{1}\right)-\mu\left(i_{1}, \ldots, i_{n}\right) & \text { if } i=i_{1}=i_{n} \\
0 & \text { otherwise. }
\end{array}\right.
$$


But the third line vanishes modulo $\Delta\left(i_{1}, \ldots, i_{n}\right)$ because of the congruence

$$
\mu\left(i_{2}, \ldots, i_{n}, i_{1}\right)-\mu\left(i_{1}, \ldots, i_{n}\right) \equiv \sum_{i=1}^{N} \int_{T_{i}} \Omega_{1 \ldots n}=\int_{\partial M} \Omega_{1 \ldots n}=\int_{M} d \Omega_{1 \ldots n}=0
$$

as in the proof of Corollary 3.3 .2 and by an application of Stokes Theorem.

\section{EXAMPLES}

4.1. Links with three components. Consider a link with three components $L_{1}$, $L_{2}$, and $L_{3}$. Pick meridians $m_{1}, m_{2}, m_{3}$ and longitudes $l_{1}, l_{2}, l_{3}$. Assume that

$$
\omega=\left(\begin{array}{cccc}
0 & \omega_{1} & \omega_{12} & 0 \\
0 & 0 & \omega_{2} & \omega_{23} \\
0 & 0 & 0 & \omega_{3} \\
0 & 0 & 0 & 0
\end{array}\right)
$$

is a connection with central curvature which is reducible with respect to $(1,2,3)$. By Proposition 3.2.1 and Lemma 3.4.4, the holonomy matrices for the $m_{i}$ 's take the form:

$$
\begin{gathered}
K_{1}=\left(\begin{array}{cccc}
0 & 1 & a_{12} & a_{123} \\
0 & 0 & 0 & 0 \\
0 & 0 & 0 & 0 \\
0 & 0 & 0 & 0
\end{array}\right), \quad K_{2}=\left(\begin{array}{cccc}
0 & 0 & b_{12} & b_{123} \\
0 & 0 & 1 & b_{23} \\
0 & 0 & 0 & 0 \\
0 & 0 & 0 & 0
\end{array}\right), \\
K_{3}=\left(\begin{array}{cccc}
0 & 0 & 0 & c_{123} \\
0 & 0 & 0 & c_{23} \\
0 & 0 & 0 & 1 \\
0 & 0 & 0 & 0
\end{array}\right) .
\end{gathered}
$$

One easily computes the only nonzero products of the $K_{i}$ 's to be:

$$
\begin{gathered}
K_{1} K_{2}=\left(\begin{array}{cccc}
0 & 0 & 1 & b_{23} \\
0 & 0 & 0 & 0 \\
0 & 0 & 0 & 0 \\
0 & 0 & 0 & 0
\end{array}\right), K_{2} K_{3}=\left(\begin{array}{cccc}
0 & 0 & 0 & b_{12} \\
0 & 0 & 0 & 1 \\
0 & 0 & 0 & 0 \\
0 & 0 & 0 & 0
\end{array}\right), \\
K_{1} K_{3}=\left(\begin{array}{cccc}
0 & 0 & 0 & a_{12}+c_{23} \\
0 & 0 & 0 & 0 \\
0 & 0 & 0 & 0 \\
0 & 0 & 0 & 0
\end{array}\right), K_{1} K_{2} K_{3}=\left(\begin{array}{cccc}
0 & 0 & 0 & 1 \\
0 & 0 & 0 & 0 \\
0 & 0 & 0 & 0 \\
0 & 0 & 0 & 0
\end{array}\right) .
\end{gathered}
$$

Thus the only nonzero brackets of the $K_{i}$ 's are

$$
\begin{gathered}
{\left[K_{1}, K_{2}\right]=-\left[K_{2}, K_{1}\right]=K_{1} K_{2}, \quad\left[K_{2}, K_{3}\right]=-\left[K_{3}, K_{2}\right]=K_{2} K_{3}} \\
{\left[K_{1}, K_{3}\right]=-\left[K_{3}, K_{1}\right]=K_{1} K_{3}, \quad \text { and } \quad\left[K_{1}, K_{2} K_{3}\right]=-\left[K_{3}, K_{1} K_{2}\right]=K_{1} K_{2} K_{3}}
\end{gathered}
$$


Plugging these values into the formula in Theorem 3.3.1, we obtain:

$$
\begin{aligned}
& \int_{T_{1}} \Omega=\mu(2,1)\left[K_{1}, K_{2}\right]+\mu(3,1)\left[K_{1}, K_{3}\right]+\mu(2,3,1)\left[K_{1}, K_{2} K_{3}\right] \\
& =\left(\begin{array}{cccc}
0 & 0 & \mu(2,1) & b_{23} \mu(2,1)+\left(a_{12}+c_{23}\right) \mu(3,1)+\mu(2,3,1) \\
0 & 0 & 0 & 0 \\
0 & 0 & 0 & 0 \\
0 & 0 & 0 & 0
\end{array}\right), \\
& \int_{T_{2}} \Omega=\mu(1,2)\left[K_{2}, K_{1}\right]+\mu(3,2)\left[K_{2}, K_{3}\right] \\
& =\left(\begin{array}{cccc}
0 & 0 & \mu(1,2) & -b_{23} \mu(1,2)+b_{12} \mu(3,2) \\
0 & 0 & 0 & \mu(3,2) \\
0 & 0 & 0 & 0 \\
0 & 0 & 0 & 0
\end{array}\right), \\
& \int_{T_{3}} \Omega=\mu(1,3)\left[K_{3}, K_{1}\right]+\mu(2,3)\left[K_{3}, K_{2}\right]+\mu(1,2,3)\left[K_{3}, K_{1} K_{2}\right] \\
& =\left(\begin{array}{cccc}
0 & 0 & 0 & -b_{12} \mu(2,3)-\left(a_{12}+c_{23}\right) \mu(1,3)-\mu(1,2,3) \\
0 & 0 & 0 & -\mu(2,3) \\
0 & 0 & 0 & 0 \\
0 & 0 & 0 & 0
\end{array}\right) .
\end{aligned}
$$

By central curvature all the entries, except for the upper right-hand corner, are zero. Thus

$$
\begin{gathered}
\mu(2,1)=\mu(1,2)=\mu(3,2)=\mu(2,3)=0, \\
\int_{T_{1}} \Omega_{123}=\left(a_{12}+c_{23}\right) \mu(3,1)+\mu(2,3,1), \\
\int_{T_{2}} \Omega_{123}=0, \\
\int_{T_{3}} \Omega_{123}=-\left(a_{12}+c_{23}\right) \mu(1,3)-\mu(1,2,3) .
\end{gathered}
$$

These calculations show the necessity of the vanishing of the linking numbers of $L_{1}$ and $L_{2}$ and of $L_{2}$ and $L_{3}$ for a reducible connection with central curvature associated to $(1,2,3)$ to exist. The next proposition shows the sufficiency of this condition.

Proposition 4.1.1. Assume that the linking numbers of $L_{1}$ and $L_{2}$ and of $L_{2}$ and $L_{3}$ are zero. Then there is a connection with central curvature which is reducible for the multi-index $(1,2,3)$ whose holonomy matrix entries $a_{12}, b_{12}, b_{23}$, and $c_{23}$ take on any prescribed real values. In particular we can produce connections with integral or nonintegral holonomy.

Proof. Choose closed 1-forms $\omega_{i}$ in $S^{3} \backslash L_{i}$ dual to $L_{i}$. Since the linking numbers of $L_{1}$ with $L_{2}$ and of $L_{2}$ with $L_{3}$ are zero, $\omega_{1} \wedge \omega_{2}$ and $\omega_{2} \wedge \omega_{3}$ are exact. Therefore one can choose 1-forms $\omega_{12}$ and $\omega_{23}$, in the complements of $L_{1} \cup L_{2}$ and $L_{2} \cup L_{3}$ respectively, with $d \omega_{12}+\omega_{1} \wedge \omega_{2}=0$ and $d \omega_{23}+\omega_{2} \wedge \omega_{3}=0$. For more details, see for example [11, Section 2.3]. This shows there is at least one connection $\omega$ with central curvature which is reducible with respect to the multi-index $(1,2,3)$. 
We next enumerate five simple modifications of a reducible connection $\omega$ with central curvature $\Omega$ which produce new reducible connections $\omega^{\prime}$ with central curvature $\Omega^{\prime}$ and describe how each modification affects holonomy and curvature. These formulas are verified by short calculations. The holonomy calculations use (2.10) and [7, Proposition 1.3, p. 252]. In the formulas below, $*$ is the base point of the fundamental group.

(1) Let $f_{1}$ be a smooth function on $S^{3} \backslash L_{1}$. Replace $\omega_{1}$ by $\omega_{1}+d f_{1}$ and $\omega_{12}$ by $\omega_{12}-f_{1} \omega_{2}$ and leave all other entries unchanged. The new curvature and holonomy satisfy: $\Omega_{123}^{\prime}=\Omega_{123}+d\left(f_{1} \omega_{23}\right), a_{12}^{\prime}=a_{12}, b_{12}^{\prime}=b_{12}-f_{1}(*)$, $b_{23}^{\prime}=b_{23}$, and $c_{23}^{\prime}=c_{23}$.

(2) Let $f_{2}$ be a smooth function on $S^{3} \backslash L_{2}$. Replace $\omega_{2}$ by $\omega_{2}+d f_{2}, \omega_{12}$ by $\omega_{12}+f_{2} \omega_{1}, \omega_{23}$ by $\omega_{23}-f_{2} \omega_{3}$ and leave all other entries unchanged. Then $\Omega_{123}^{\prime}=\Omega_{123}, a_{12}^{\prime}=a_{12}+f_{2}(*), b_{12}^{\prime}=b_{12}, b_{23}^{\prime}=b_{23}$, and $c_{23}^{\prime}=c_{23}-f_{2}(*)$.

(3) Let $f_{3}$ be a smooth function on $S^{3} \backslash L_{3}$. Replace $\omega_{3}$ by $\omega_{3}+d f_{3}$ and $\omega_{23}$ by $\omega_{23}+f_{3} \omega_{2}$ and leave all other entries unchanged. Then $\Omega_{123}^{\prime}=\Omega_{123}-$ $d\left(f_{2} \omega_{23}\right), a_{12}^{\prime}=a_{12}, b_{12}^{\prime}=b_{12}, b_{23}^{\prime}=b_{23}+f_{3}(*)$, and $c_{23}^{\prime}=c_{23}$.

(4) Let $\phi_{12}$ be a closed 1 -form on $S^{3} \backslash\left(L_{1} \cup L_{2}\right)$. Replace $\omega_{12}$ by $\omega_{12}+\phi_{12}$, and leave all other entries unchanged. Then $\Omega_{123}^{\prime}=\Omega_{123}+\phi_{12} \wedge \omega_{3}, a_{12}^{\prime}=a_{12}+$ $A_{1}, b_{12}^{\prime}=b_{12}+A_{2}, b_{23}^{\prime}=b_{23}$, and $c_{23}^{\prime}=c_{23}$, where $\left[\phi_{12}\right]=A_{1}\left[\omega_{1}\right]+A_{2}\left[\omega_{2}\right]$ in $H^{1}\left(S^{3} \backslash\left(L_{1} \cup L_{2}\right)\right)$.

(5) Let $\phi_{23}$ be a closed 1-form on $S^{3} \backslash\left(L_{2} \cup L_{3}\right)$. Replace $\omega_{23}$ by $\omega_{23}+\phi_{23}$, and leave all other entries unchanged. Then $\Omega_{123}^{\prime}=\Omega_{123}+\omega_{1} \wedge \phi_{23}, a_{12}^{\prime}=a_{12}$, $b_{12}^{\prime}=b_{12}, b_{23}^{\prime}=b_{23}+B_{2}$, and $c_{23}^{\prime}=c_{23}+B_{3}$, where $\left[\phi_{23}\right]=B_{2}\left[\omega_{2}\right]+B_{3}\left[\omega_{3}\right]$ in $H^{1}\left(S^{3} \backslash\left(L_{2} \cup L_{3}\right)\right)$.

Since the values of the $f_{i}(*)$ and of $A_{1}, A_{2}, B_{2}$, and $B_{3}$ can be chosen arbitrarily, applying the 5 modifications in succession allows one to prescibe arbitrary real values to $a_{12}, b_{12}, b_{23}$, and $c_{23}$.

Remark 4.1.2. Any two connections, $\omega$ and $\tilde{\omega}$, with central curvature which are reducible with respect to $(1,2,3)$ are related through a succession of these five modifications. First there exist functions $f_{i}$ such that $\tilde{\omega}_{i}=\omega_{i}+d f_{i}$. Thus an application of the modifications $1,2,3$ in succession produces a connection $\omega^{\prime}$ that satisfies $\omega_{i}^{\prime}=\tilde{\omega}_{i}$, for $i=1,2,3$. But then $\phi_{i j}=\tilde{\omega}_{i j}-\omega_{i j}^{\prime}$ is closed since $d \phi_{i j}=$ $d \tilde{\omega}_{i j}-d \omega_{i j}^{\prime}=-\tilde{\omega}_{i} \wedge \tilde{\omega}_{j}+\omega_{i}^{\prime} \wedge \omega_{j}^{\prime}=0$ for $i j=12$ or $i j=23$. Thus $\tilde{\omega}$ results from $\omega^{\prime}$ by applying modifications 4 and 5 . Note that the effect of modification 4 or 5 on the curvature illuminates how indeterminancy of the Massey product can happen.

4.2. Constructing connections with integral holonomy. Because of the duality between homology and cohomology, Massey products in the complement of a link are often computed using intersection theory. In this approach one utilizes a system of surfaces that are dual to the cocycles employed in a defining system for the Massey product. For example, see [5, 12. Regularization of this system, a process that replaces each surface by its Thom form, produces a nilpotent connection with central curvature and integral holonomy. Hain carried out a similar regularization in [6].

Let $L=L_{1} \cup \cdots \cup L_{N}$ be an oriented link in $S^{3}$. Let $W$ be an oriented arc or circle smoothly embedded in $S^{3}$. We say $W$ is in proper position relative to $L$ if either (a) $W \subset L$ or (b) the interior of $W$ is disjoint from $L$ and its boundary points $\partial W$ are contained in $L$. Let $V$ be a smooth, compact oriented surface embedded 
in $S^{3}$ with boundary $\partial V$ which may have corners. This means that $\partial V$ is a finite union of sections consisting of smooth arcs and/or smooth circles, a corner being a common endpoint of two arcs. Each section of $\partial V$, whether an arc or a circle, inherits an orientation from $V$ as usual. We will also consider $\partial V$ to be a 1-chain in $S^{3}$ satisfying $\partial \partial V=0$. We say $V$ is in proper position relative to $L$ if the interior of $V$ is transverse to $L$ and if each section of the boundary is in proper position relative to $L$.

We say two properly positioned surfaces $V$ and $V^{\prime}$ relative to $L$ intersect properly if they intersect transversely in finitely many arcs and circles which are themselves in proper position relative to $L$. The orientations of $V$ and $V^{\prime}$ determine the orientation of $V \cap V^{\prime}$ according to the usual convention. We will also regard $V \cap V^{\prime}$ as a 1 -chain in $S^{3}$.

A defining system corresponding to a given multi-index $\left(i_{1}, \ldots, i_{n}\right)$ consists of an array of properly positioned surfaces relative to $L$ :

$$
\begin{array}{ccccc}
V_{1} & V_{12} & \cdots & V_{12 \ldots n-1} & \\
& V_{2} & \cdots & V_{2 \ldots n-1} & V_{2 \ldots n} \\
& & \ddots & \vdots & \vdots \\
& & & V_{n-1 n} & V_{n-1} \\
& & & & V_{n}
\end{array}
$$

such that

$$
\partial V_{1}=L_{i_{1}}, \partial V_{2}=L_{i_{2}}, \ldots, \partial V_{n}=L_{i_{n}}
$$

and for every subscript $i \ldots j \neq 1 \ldots n$,

$$
\partial V_{i \ldots j}+V_{i} \cap V_{i+1 \ldots j}+\cdots+V_{i \ldots j-1} \cap V_{j}=0 \text { rel } L .
$$

The "curvature" of (4.1) is defined by the formula

$$
R_{1 \ldots n}=V_{1} \cap V_{2 \ldots n}+\cdots+V_{1 \ldots n-1} \cap V_{n} .
$$

The assumption that only proper intersections occur in (4.2) and (4.3) is included in the definition of a defining system.

Let us recall the definitions of the Thom forms of compact, oriented submanifolds 10, 1, 6. Given $\epsilon>0$, let $N_{\epsilon}(A)$ denote the $\epsilon$-neighborhood of the subset $A \subset S^{3}$.

(1) An $\epsilon$-Thom form of a point $p$ is a smooth 3-form $\tau$ on $S^{3}$ satisfying

(a) the support of $\tau$ is contained in $N_{\epsilon}(p)$,

(b) $d \tau=0$, and

(c) $\int_{D} \tau=1$, where $D$ is the $\epsilon$-ball centered at $p$.

(2) An $\epsilon$-Thom form of an arc (or circle) $W$ is a smooth 2 -form $\phi$ on $S^{3}$ satisfying

(a) the support of $\phi$ is contained in $N_{\epsilon}(W)$,

(b) $d \phi=\tau_{1}-\tau_{0}$, where for $i=0,1, \tau_{i}$ is an $\epsilon$-Thom form of the endpoint $W(i)$ of $W$. (This equation becomes $d \phi=0$ when $W$ is a circle.)

(c) $\int_{D} \phi=1$ for every normal oriented $\epsilon$-disk $D$ centered at a point of $W \backslash N_{\epsilon}(\partial W)$.

(3) An $\epsilon$-Thom form of a surface $V$ is a smooth 1-form $\omega$ on $S^{3}$ satisfying

(a) the support of $\omega$ is contained in $N_{\epsilon}(V)$,

(b) $d \omega=\sum \phi_{i}$, where $\phi_{i}$ is an $\epsilon$-Thom form of the $i$-th section of $\partial V$, and

(c) $\int_{D} \omega=1$ for every normal oriented $\epsilon$-arc $D$ centered at a point of $V \backslash N_{\epsilon}(\partial V)$. 
Theorem 4.2.1. Given a defining system (4.1) and a sufficiently small $\epsilon>0$, there exists a connection $\omega$ on $S^{3}$ such that (i) for each $i \ldots j, \omega_{i \ldots j}$ is an $\epsilon-T h o m$ form for $V_{i \ldots j}$, (ii) the curvature form $\Omega$ of $\omega$ is supported in $N_{\epsilon}\left(L \cup R_{1 \ldots n}\right)$, and (iii) if $i \ldots j \neq 1 \ldots n$, then $\Omega_{i \ldots j}$ is supported in $N_{\epsilon}(L)$.

Thus the curvature is central when $\omega$ is restricted to the complement of $N_{\epsilon}(L)$. Furthermore $\omega$ has integral holonomy in this complement $S^{3} \backslash N_{\epsilon}(L)$.

Remark 4.2.2. A connection $\omega$ with the properties listed in this theorem is called an $\epsilon$-regularization of the system (4.1). For sufficiently small $\epsilon$, the complements $S^{3} \backslash L$ and $S^{3} \backslash N_{\epsilon}(L)$ are diffeomorphic. Thus regularization produces connections in $S^{3} \backslash L$ with central curvature and integral holonomy.

To sketch the proof, we need three lemmas.

Lemma 4.2.3. Given a properly positioned surface $V$ and any sufficiently small $\epsilon$, there exists an $\epsilon$-Thom form of $V$.

Proof. It is convenient to use the properties of the regularization operator $R$ for currents listed in [14, Théorème 12]. By regarding $V$ as a de Rham current, $R(V)$ is the required Thom form. Property (2) of $R$ immediately implies (3)(a). The linearity of $R$ and the fact that it intertwines the boundary operator on currents with the exterior derivative on forms makes the verification of (3)(b) easy. Property (1) of $R$ leads to (3)(c).

The next two lemmas can be proved by standard arguments [10, 1].

Lemma 4.2.4. Given two $\epsilon$-Thom forms $\phi_{1}$ and $\phi_{2}$ of the arc (or the circle) $W$, there exists a smooth 1 -form $\psi$ with support in $N_{\epsilon}(W)$ such that $\phi_{2}=\phi_{1}+d \psi+\xi$, where $\xi$ is a 2 -form supported in $N_{\epsilon}(\partial W)$. ( $\xi=0$ when $W$ is a circle.)

Lemma 4.2.5. Let $V$ and $V^{\prime}$ be two properly positioned surfaces which intersect properly. For sufficiently small $\epsilon>0$, if $\omega$ and $\omega^{\prime}$ are $\epsilon$-Thom forms of $V$ and $V^{\prime}$ respectively, then $\omega \wedge \omega^{\prime}$ is a sum of $\epsilon$-Thom forms of the components of $V \cap V^{\prime}$.

Proof of Theorem 4.2.1. The $\omega_{i \ldots j}$ are constructed recursively. Start by using Lemma 4.2 .3 to pick Thom forms $\omega_{1}, \ldots, \omega_{n}$ for $V_{1}, \ldots, V_{n}$. Suppose the forms have been chosen for all the surfaces below the level of $V_{i \ldots j}$. Choose a Thom form $\omega^{\prime}$ for $V_{i \ldots j}$ by Lemma 4.2.3. We will modify $\omega^{\prime}$ in an $\epsilon-$ neighborhood of each section $W$ of $\partial V_{i \ldots j}$ that cancels with a component of one of the intersections in (4.2). Each such section $W$ has two Thom forms, the first, $\phi_{1}$, coming via (3)(b) from $d \omega^{\prime}$, and the second, $\phi_{2}$, coming via Lemma 4.2 .5 from the wedge product of previously constructed Thom forms of surfaces from lower levels in the system. Lemma 4.2.4 produces a 1-form $\psi$ supported in $N_{\epsilon}(W)$, such that $\omega^{\prime}+\psi$ is still a Thom form for $V_{i \ldots j}$ and such that $d\left(\omega^{\prime}+\psi\right)$ equals $\phi_{2}$ in $N_{\epsilon}(W) \backslash N_{\epsilon}(\partial W)$. Therefore, by adding the $\psi$ 's for each of the $W$ 's to $\omega^{\prime}$ and taking into account that $N_{\epsilon}(\partial W) \subset N_{\epsilon}(L)$, there results a Thom form $\omega_{i \ldots j}$ for $V_{i \ldots j}$ such that

$$
\Omega_{i \ldots j}=d \omega_{i \ldots j}+\omega_{i} \wedge \omega_{i+1 \ldots j}+\cdots+\omega_{i \ldots j-1} \wedge \omega_{j}
$$

has support in $N_{\epsilon}(L)$. Set $\omega_{1 \ldots n}=0$. By construction, the support of

$$
\Omega_{1 \ldots n}=\omega_{1} \wedge \omega_{2 \ldots n}+\omega_{12} \wedge \omega_{3 \ldots n}+\cdots+\omega_{1 \ldots n-1} \wedge \omega_{n}
$$

is contained in $N_{\epsilon}\left(L \cup R_{1 \ldots n}\right)$. 
We now show that $\omega$ restricted to the complement of $N_{\epsilon}(L)$ has integral holonomy. Any loop $\gamma$ in $S^{3} \backslash N_{\epsilon}(L)$ is homotopic to a loop $\tilde{\gamma}$ in the complement of

$$
N_{\epsilon}\left(R_{1 \ldots n}\right) \cup N_{\epsilon}\left(\bigcup_{i \ldots j} \partial V_{i \ldots j}\right)
$$

that meets each surface $V_{i \ldots j}$ transversely. The integral of $\omega_{i \ldots j}$ along $\tilde{\gamma}$ equals the intersection number of $\tilde{\gamma}$ with $V_{i \ldots j}$ by $(3)(\mathrm{c})$ in the definition of the Thom form. Likewise the iterated line integrals in (2.10) count the number of iterated intersections. See the discussion in [6, pp. 46-48]. Thus all entries of the holonomy matrix $U(\tilde{\gamma})$ are integers. Applying Corollary 2.3 .2 to the contractible curve $\gamma \tilde{\gamma}^{-1}$ in $S^{3} \backslash N_{\epsilon}(L)$ implies that

$$
U(\gamma) U(\tilde{\gamma})^{-1}=I+X,
$$

where $X \in \mathfrak{J}^{n}$. Thus $U(\gamma)=(I+X) U(\tilde{\gamma})$ has integer entries except possibly for the $1 \ldots n$ entry.

The following example will provide a counterexample to Theorem 3.1 and Theorem 3.2 in [11].

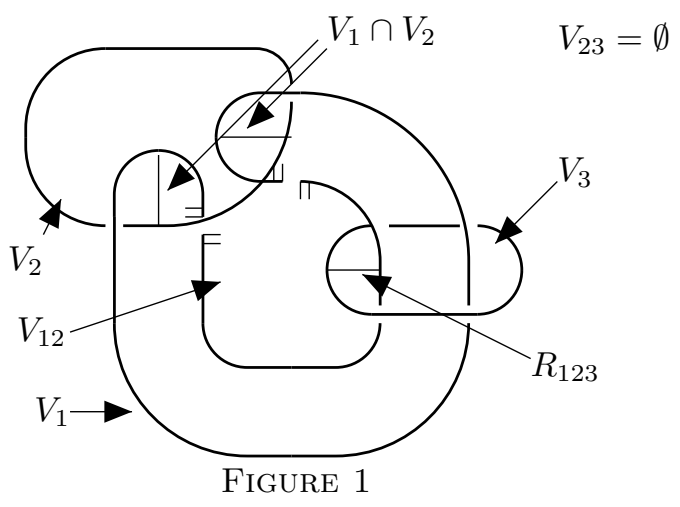

Figure 1 pictures a system of properly positioned surfaces for the Borromean rings which can be used to compute the Massey product $\left\langle\alpha_{1}, \alpha_{2}, \alpha_{3}\right\rangle$. (The surfaces are oriented so that their boundary curves are oriented counterclockwise when viewed in the figure. The system corresponds to the multi-index $(1,2,3)$.) Since $V_{2} \cap V_{3}=\emptyset$ and $V_{23}=\emptyset$, an $\epsilon$-regularization of the system produces a connection with $\omega_{23}=0$ and $\Omega_{23}=d \omega_{23}+\omega_{2} \wedge \omega_{3}=0$ as forms on $S^{3}$. The Massey number $\ell_{123}=\int_{T_{1}} \Omega_{123}$ can be computed as the intersection number of the $\epsilon$-torus $T_{1}$ about $L_{1}$ with $R_{123}$. Thus $\ell_{123}=1$ because $R_{123}$, a single arc from $L_{1}$ to $L_{3}$, intersects $T_{1}$ once positively. Equation (3.8) of [11, Theorem 3.1, p. 713] translated into our notation thus asserts that

$$
1=\ell_{123}=\int_{S^{3}} \Omega_{1} \wedge \omega_{23} .
$$

But the integral is 0 since $\omega_{23}=0$. The use of equation (3.9) [11, p. 714] in the proof, which incorrectly leaves out some terms from Bianchi's identity, apparently accounts for the mistake. 
The assertion of [11, Theorem 3.2] that, in computing the holonomy of the longitude $l_{1}$ of $L_{1}$, all the iterated integrals but the first vanish, does not hold in this example. Precisely, taking $n=3$ as in our example, equation (3.12) [11, p. 716] asserts incorrectly that

$$
\ell_{123}=\int_{l_{1}} \omega_{23}=\int_{l_{1}} \omega_{23}+\iint_{l_{1}} \omega_{2} \omega_{3}
$$

since $\ell_{123}=1, \int_{l_{1}} \omega_{23}=0$, and the doubly iterated integral $\iint_{l_{1}} \omega_{2} \omega_{3}=1$.

\section{REFERENCES}

[1] R. Bott and L. Tu. Differential Forms in Algebraic Topology, Springer-Verlag, New YorkHeidelberg-Berlin, 1982. MR658304 (83i:57016)

[2] K.-T. Chen. Connections, holonomy and path space homology. Proceedings of Symposia in Pure Mathematics 27, Part 1 (1975) 39-52. MR0440540 (55:13414)

[3] K.-T. Chen. Extension of $C^{\infty}$ function algebra by integrals and Malcev completion of $\pi_{1}$, Advances in Math. 23 (1977) 181-210. MR0458461 (56:16664)

[4] K.-T. Chen. Iterated path integrals, Bull. Amer. Math. Soc. 83 (1977) 831-879. MR:0454968(56:13210)

[5] R. Fenn. Techniques of geometric topology, London Mathematical Society Lecture Note Series, 57, Cambridge University Press, Cambridge, 1983. MR787801 (87a:57002)

[6] R. Hain. Iterated integrals, intersection theory and link groups, Topology 24 (1985) 45-66. Erratum, Topology 25 (1986) 585-586. MR790675 (86m:57008) MR862442 (87m:57005)

[7] R. Hain. The geometry of the mixed Hodge structure on the fundamental group, Proceedings of Symposia in Pure Mathematics 46, Part 2 (1987) 247-282. MR927984 (89g:14010)

[8] S. Kobayashi and K. Nomizu. Foundations of Differential Geometry, Vol. I, Interscience Publishers-John Wiley \& Sons, New York and London, 1963. MR0152974 (27:2945)

[9] T. Levi-Civita. The Absolute Differential Calculus, Dover Publications, Inc., New York, 1977. MR.2422211 (2011b:53031)

[10] T. Nagano. Homotopy Invariants in Differential Geometry, American Mathematical Society, Providence, 1970. MR0268906 (42:3803)

[11] V. Penna and M. Spera. Higher order linking numbers, curvature and holonomy, Journal of Knot Theory and Its Ramifications, 11 (2002) 701-723. MR1918809 (2003g:57010)

[12] R. Porter. Milnor's $\bar{\mu}$-invariants and Massey products, Trans. Amer. Math. Soc. 257 (1980) 39-71. MR.549154 (81a:57021)

[13] H. Reckziegel and E. Wilhelmus. How the curvature generates the holonomy of a connection in an arbitrary fibre bundle, Result. Math. 49 (2006) 339-359. MR.2288249 (2007k:53068)

[14] G. de Rham. Variétés différentiables, Hermann, Paris, 1960.

[15] L. Schlesinger. Parallelverschiebung und Krümmungstensor, Math. Ann. 99 (1928) 413434. MR 1512459

[16] C. Teleman. Généralisation du groupe fondamental, Annales Scientifiques de l'É.N.S 77 (1960) 195-234. MR0124068 (23:A1385c)

[17] J. Taveres. Chen integrals, generalized loops and loop calculus, Intl. J. Mod. Phys. A 9 (1994) 4511-4548. MR1295759 (95i:58021)

[18] V. Turaev. The Milnor invariants and Massey products. Zap. Naučn. Sem. Leningrad. Otel. Mat. Inst. Steklov. (LOMI) 66 (1976) 189-203, 209-210. (Russian) Translation in: Journal of Soviet Mathematics 12 (1979) 128-137. MR0451251 (56:9538)

Department of Mathematics and Computer Science, Saint Louis University, St. Louis, Missouri 63103

E-mail address: hebdajj@slu.edu

Department of Mathematics and Computer Science, Saint Louis University, St. Louis, Missouri 63103

E-mail address: tsaumc@slu.edu 